\title{
Exotic Solutions to the Solar Neutrino Anomaly
}

\author{
M. M. Guzzo \\ Instituto de Física Gleb Wataghin \\ Universidade Estadual de Campinas, UNICAMP \\ 13083-970, Campinas, SP, Brazil
}

Received on 19 April, 2001

\begin{abstract}
We analyze the status of the exotic solutions to the solar neutrino problem, i.e., those solutions based on new phenomena which are not the usual neutrino oscillations induced by masses and mixing. These solutions are based on different assumptions: a) resonant spin-flavor precession induced by non-vanishing neutrino magnetic moment, b) the existence of non-standard flavor-changing and non-universal neutrino interactions and c) the violation of the equivalence principle. We investigate the quality of the fit provided by each one of these solutions not only to the total rate measured by all solar neutrino experiments but also to the day-night and seasonal variations of the event rate, as well as the recoil electron energy spectrum measured by the SuperKamiokande collaboration.
\end{abstract}

\section{Introduction}

Homestake [1], GALLEX/GNO [2], SAGE [3], Kamiokande [4] and SuperKamikande [5] have observed a solar neutrino flux which is smaller than predicted by the standard solar models (SSM) $[6,7,8,9,10]$. This discrepancy has been called the solar neutrino problem [11]. Better statistics and calibration of the pioneering experiments, as well as the first nextgeneration experiment SuperKamiokande, measuring the solar neutrino spectrum and the event rate as a function of the zenith angle with unprecedented precision, have provided a lot of new information about the solar neutrino problem [11]. On the theoretical side several substantial improvements have been made in the SSM which now includes diffusion of helium and heavy elements and updated low energy nuclear cross sections relevant to the solar neutrino production [12]. Furthermore, the SSM has received an important independent confirmation by the excellent agreement between its predicted sound speeds and recent helioseismological observations [7].

In order to understand the solar neutrino anomaly it has been suggested that neutrinos are endowed with properties which are not present in the standard electroweak theory [13]. These new properties allow the electron neutrinos to be converted along their way from the center of the sun to the detectors on earth into different neutrino flavors, i.e. into muon, tau, or possibly sterile [14] neutrinos. The fact that the terrestrial experiments are less sensitive to these neutrino flavors explains the observed lower counting rates.

Several mechanisms can provoke the electron neutrino convertion into different neutrino flavors. Up to our knowledge, the mechanisms that fit the solar neutrino data can be classified in four essentially different types:

The most famous solutions to the solar neutrino anomaly assume that neutrinos are massive and there is mixing in the lepton sector. Under this circunstance, neutrino flavor oscillations can happen in vacuum [15] as well as in matter where it can be resonantly enhanced [16, 17] (the Mikheyev-Smirnov-Wolfenstein (MSW) effect). In both scenarios solar electron neutrinos can be converted into neutrino of a different flavor (muon or tau) and consequently explain the deficit of observed solar neutrino deficit to the predictions of the SSM. These solutions are called the standard solutions to the solar neutrino problem and are very well described in recent references [18], [19] and [20].

In this review, we will not describe the solutions to the solar neutrino anomaly based on mass-induced oscillation phenomenon. We wil concentrate on more exotic solution like those one described below.

\section{Resonant spin-flavor phe- nomenon}

Assuming a nonvanishing transition magnetic moment of neutrinos, active solar neutrinos interacting with the magnetic field in the Sun can be spin-flavor converted into sterile nonelectron neutrinos [21,22] (if we are dealing with Dirac particles) or into active nonelectron antineutrinos [23] (if the involved particles are Majorana). In both cases the resulting particles interact with solar neutrino detectors significantly less than the original active electron neutrinos in such a way that this phe- 
nomenon can induce a depletion in the detectable solar neutrino flux. Spin-flavor precession of neutrinos can be resonantly enhanced in matter [24, 25], in close analogy with the MSW effect $[16,17]$. In this case the precession strongly depends on the neutrino energy and provokes different suppressions for each portion of the solar neutrino energy spectrum. Therefore RSFP provides a satisfactory description $[26,27,28,29,30,31,32]$ of the actual experimental panorama $[1,2,3,4,5]$ : all experiments detect less than the theoretically predicted solar neutrino fluxes $[9,10]$ and different suppressions are observed in each experiment, suggesting that the mechanism to conciliate theoretical predictions and observations has to differentiate the different parts of the solar neutrino spectrum.

The time evolution of neutrinos interacting with a magnetic field $B$ through a nonvanishing neutrino magnetic moment $\mu_{\nu}$ in matter is governed by a Schrödinger-like equation [24, 25];

$$
i \frac{d}{d t}\left(\begin{array}{c}
\nu_{e_{L}} \\
\bar{\nu}_{\mu_{R}}
\end{array}\right)=\left(\begin{array}{cc}
a_{\nu_{e}} & \mu_{\nu} B \\
\mu_{\nu} B & \frac{\Delta m^{2}}{2 E}+a_{\nu_{\mu}}
\end{array}\right)\left(\begin{array}{c}
\nu_{e_{L}} \\
\bar{\nu}_{\mu_{R}}
\end{array}\right),
$$

where $\nu_{e}$ and $\bar{\nu}_{\mu_{R}}$ are active electron neutrinos and muon antineutrinos, respectively, $\Delta m^{2}=m_{\nu_{\mu}}^{2}-m_{\nu_{e}}^{2}$ is their squared mass difference and $E$ is the neutrino energy, $a_{\nu_{e}}=G_{F}\left(2 N_{e}-N_{n}\right) / \sqrt{2}$ and $a_{\nu_{\mu}}=G_{F} N_{n} / \sqrt{2}$, with $N_{e}$ and $N_{n}$ being electron and neutron number densities, respectively. In eq. (1) it is assumed that neutrinos are Majorana particles. For the Dirac case, the spin-flavor precession involves $\nu_{e} \leftrightarrow \nu_{s}$, where $\nu_{s}$ is a sterile neutrino and $a_{\nu_{s}}=0$.

In order to obtain the survival probability one should integrate the evolution equations (1) with varying matter density in the Sun [11] for some assumed profiles of the magnetic field which will be described below. Using the solar neutrino flux in ref. [8], it was computed in Ref. [32] the expected solar neutrino event rate in each experiment, taking into account the relevant absorption cross sections [11] for ${ }^{71} \mathrm{Ga}$ and ${ }^{37} \mathrm{Cl}$ experiments as well as the scattering cross sections for $\nu_{e} e^{-}$and $\bar{\nu}_{\mu}-e^{-}$reactions including also the efficiency function for the SuperKamiokande experiment in the same way as in ref. [33]. Note that in this analysis it was always adopted the solar model in ref. [8] as a reference SSM.

It is obvious from the evolution equations (1) that the RSFP mechanism crucially depends on the solar magnetic field profile along the neutrino trajectory. In the analisys of Ref. [32] it was chosen several different profiles which cover in general all the previously $[27,28,29,30,31]$ analysed magnetic profiles which led to a solution to the solar neutrino anomaly. In Fig. 1, these magnetic fields are presented in their general aspects. The constant magnetic profile $B_{1}(r)$ was adopted in references [29], while the general aspects of the profiles $B_{3}(r)$ and $B_{4}(r)$ have already appeared in refs. [28, 30, 27], and [31], respectively.

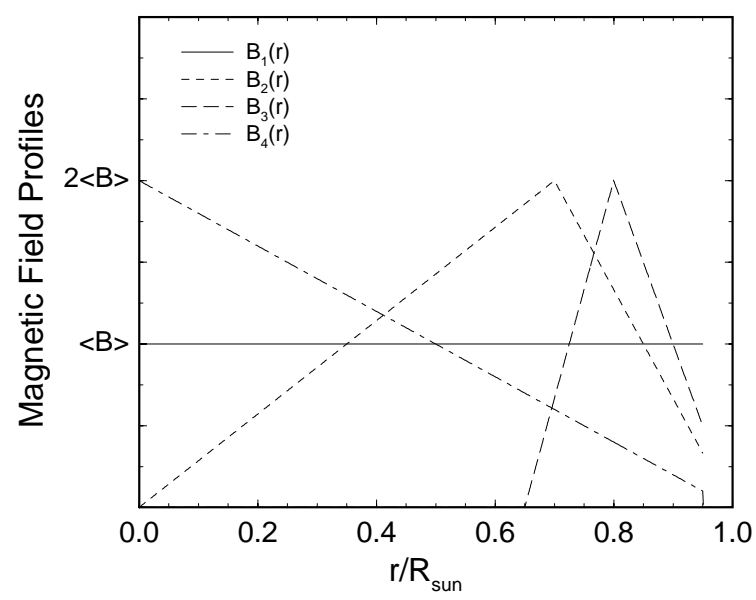

Figure 1. Various magnetic field profiles used in this work. For each field $\langle B\rangle$ is defined as the average of the field over the region where $B(r)$ is not zero.

Note that close to the solar surface $\left(r>0.95 R_{\odot}\right)$ the magnetic field was switched off for all the profiles. $\chi^{2}$ is defined as follows,

$$
\chi^{2}=\sum_{i, j}\left(R_{i}^{t h}-R_{i}^{o b s}\right)\left[\sigma_{i j}^{2}(\mathrm{tot})\right]^{-1}\left(R_{j}^{t h}-R_{j}^{o b s}\right),
$$

where $(i, j)$ run through three experiments, i.e., ${ }^{71} \mathrm{Ga}$, ${ }^{37} \mathrm{Cl}$ and SuperKamiokande, and the total error matrix $\sigma_{i j}^{2}$ (tot) and the expected event rates $R_{i}$ are computed as follows. It was essentially used the procedure of Ref. [34] for the derivation of the error matrix and to describe the correlations of errors we used in this work.

Including now the experimental observations on the solar neutrino signal it is possible to determine the region in the $\Delta m^{2}-\langle B\rangle$ parameter space which leads to a RSFP solution to the solar neutrino problem for a specified confidence level. It is presented the $\Delta m^{2}-\langle B\rangle$ parameter region which can account for all the solar neutrino data, at 90, 95 and 99\% C. L. in Figs. 2(a), (b), (c) and (d), for the magnetic profiles $B_{1}(r), B_{2}(r)$, $B_{3}(r)$ and $B_{4}(r)$, respectively.

From Figs. 2 (a) to (d) one observes that a solution to the solar neutrino problem can be found when $\langle B\rangle \gtrsim$ few times $10 \mathrm{kG}$ and $\Delta m^{2}$ is the order of $10^{-8}$ to $10^{-7} \mathrm{eV}^{2}$ for any of the magnetic profiles used in this work. Nevertheless, the quality of the fit, measured by the minimum $\chi^{2}$ criterion, varies a lot. The poorest fit is obtained when the continuously decaying magnetic field profile $B_{4}$ is used, with $\chi_{\min }^{2}=6.1$ for one (three data points - two free parameters) degrees of freedom. Better fits are obtained when the $B_{1}$ (uniform) and $B_{2}$ (large triangle) fields are employed showing $\chi_{\text {min }}^{2}=2.0$ and 1.8, respectively. And the best fit appears when the triangular field in the solar convective zone, $B_{3}$, is employed, with a rather small value $\chi_{\text {min }}^{2}=0.13$. For 
this profile, we note that, as expected from Fig. 3 (c) we have several local best fit points also indicated in Fig. 4 (c) by the open circles whose corresponding $\chi_{\min }^{2}$ are, from left to right, 2.3, 0.29 and 0.19 .
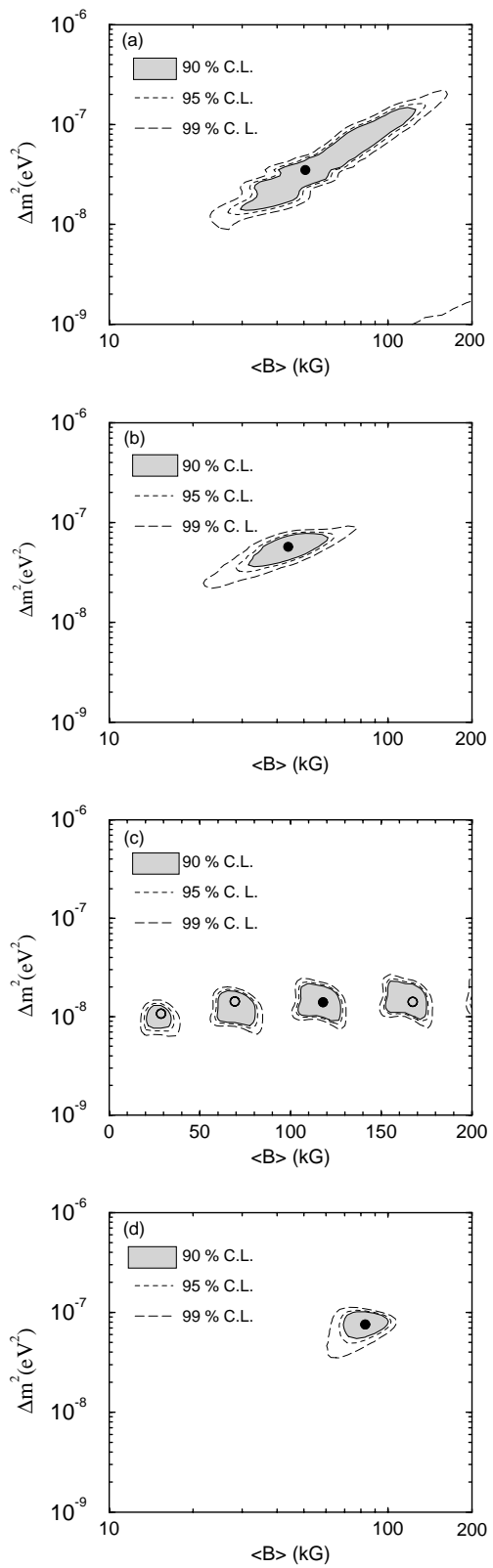

Figure 2. The Allowed RSFP solution to the solar neutrino problem. The parameter region allowed at 90,95 and 99 $\%$ C. L. are shown in (a), (b), (c) and (d) for the magnetic field profiles, $B_{1}, B_{2}, B_{3}$ and $B_{4}$, respectively, sketched in Fig. 1. We indicate best fit points by filled circles. In (c) we also indicate, by the open circles, the local best fit points inside each island delimited by $90 \%$ C.L. curves.

The reason why a very good fit is obtained for $B_{3}$ is that this profile can provide the required suppression patterns of various neutrino flux implied by latest the data [35] as discussed in ref. [30]. First note that low energy $p p$ neutrinos are not so suppressed because the resonance positions are located in the inner region where the magnetic field is zero or small. However, intermediate energy ${ }^{7} \mathrm{Be}$ neutrinos can be strongly suppressed due to the rapid increase of the field at the bottom of the convective zone since their resonance position is in a slightly outer region than the $p p$ one. On the other hand, high energy ${ }^{8} \mathrm{~B}$ neutrinos are moderately suppressed because their resonance positions are closer to the solar surface than the ${ }^{7} \mathrm{Be}$ ones, where the field is decreasing.

The best fitted values of $\langle B\rangle$ and $\Delta m^{2}$ as well as $\chi_{m i n}^{2}$ obtained from these different profiles are summarized in Table $\mathrm{I}$.

\begin{tabular}{cccc}
\hline \hline Profile & $\langle B\rangle(\mathrm{kG})$ & $\Delta m^{2}\left(10^{-8} \mathrm{eV}^{2}\right)$ & $\chi_{\min }^{2}$ \\
\hline 1 & $50.6(40.9)$ & $3.5(2.3)$ & $2.0(6.2)$ \\
2 & $47.1(40.8)$ & $6.1(4.6)$ & $1.8(5.7)$ \\
3 & $118(69.4)$ & $1.5(1.2)$ & $0.13(1.3)$ \\
4 & $82.9(81.6)$ & $8.1(6.6)$ & $6.1(11.4)$ \\
\hline
\end{tabular}

TABLE I. The best fitted parameters and $\chi_{m i n}^{2}$ for the Majorana case. Dirac case is presented in the parentheses.

The same analysis for the Dirac neutrino case was done. The corresponding plots for the allowed region are not shown here since they are rather similar to what have been presented above, if the same magnetic field profile is assumed. Instead, for the case of Dirac neutrinos, we only present the best fitted parameters and $\chi_{\min }^{2}$ in the parentheses in Table I. We see from this table that, the Dirac case always leads to a worse fit if the same magnetic field profile is assumed. To understand this we should note that for the Dirac case, $\nu_{e}$ 's are converted into the right handed muon (or tau) neutrino, which do not contribute to any of the solar experiments including the water Cherenkov experiment [36]. This makes it difficult to conciliate the difference between the SuperKamiokande and ${ }^{37} \mathrm{Cl}$ data. In contrast in the Majorana case, converted right handed neutrino $\bar{\nu}_{\mu}$ 's do contribute to the signal observed in the SuperKamiokande detector.

Let me now briefly comment about the possibility of having such strong magnetic field in the Sun. While there is no generally accepted theory of solar magnetic field, it is possible to bound the field strength from very general arguments. It can be shown [11] that the magnetic field less than $10^{6} \mathrm{kG}$ in the solar core or less than $10^{4} \mathrm{kG}$ in the solar convective zone, will hardly affect the thermal structure and nuclear reaction processes well described by the standard solar model. These values come from the requirement that the magnetic pressure should be much smaller than the gas pressure, and can be regarded as the most generous upper limits of the magnetic field inside the Sun. More stringent bounds on the magnetic field in the convective zone are found in refs. [37, 38] where the discussion is based on the non-linear effects which eventually prevents the growth of magnetic fields created by the dynamo process. Naive limit can be obtained by estimating the 
required field tension necessary to prevent a fluid element from sinking into a magnetically stratified region, so that the magnetic flux would not be further amplified. By equating the magnetic tension to the energy excess of a sinking element at the bottom of the convective zone, Schmitt and Rosner [37] obtained $\sim 10 \mathrm{kG}$ as an upper bound for the magnetic field, which is of the order of the magnitude we need to have a good fit to the solar data by RSFP mechanism for the reference value of magnetic moment, $\mu_{\nu}=10^{-11} \mu_{B}$.

Finally, we briefly discuss how the recoil electron energy spectra in the SuperKamiokande detector will be affected by the RSFP mechanism [39].
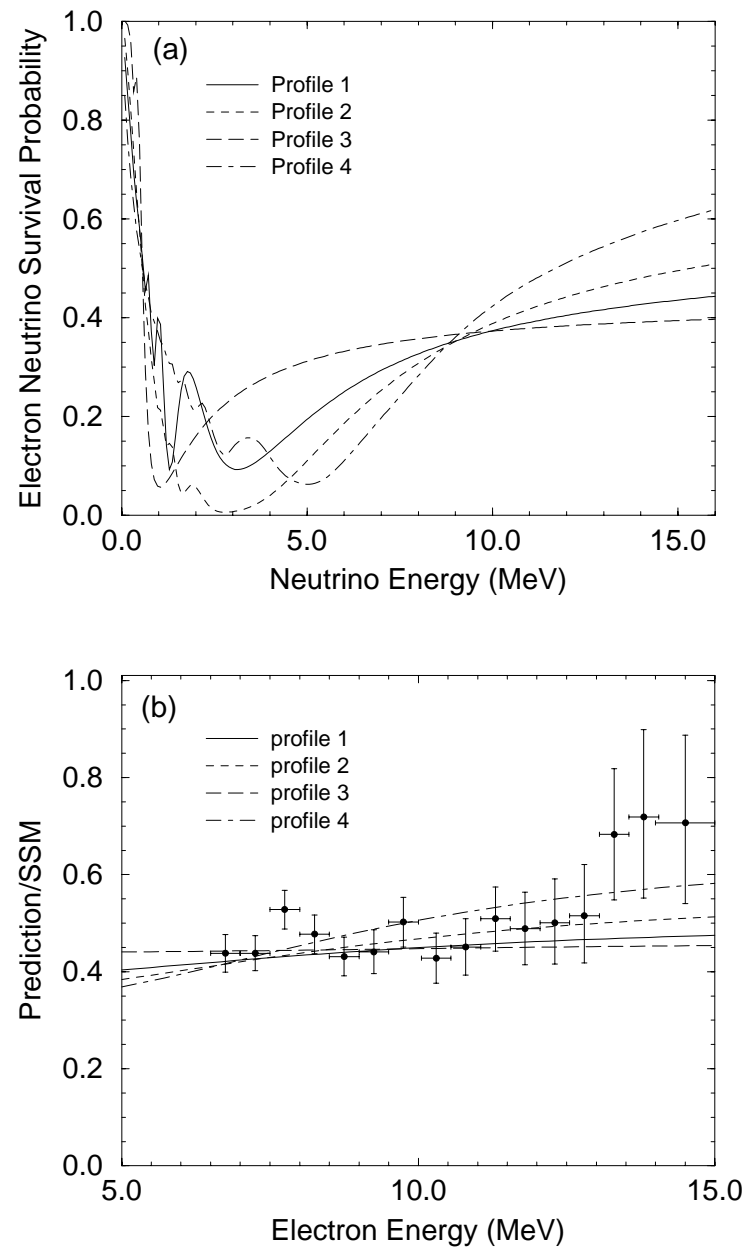

Figure 3. We plot in (a) electron neutrino survival probability as a function of energy with the best fitted parameters for various field profiles. In (b) we plot recoil electron energy spectra expected from RSFP scenario using our best fit parameters, divided by the SSM prediction. The SuperKamiokande data are also shown by the filled circles with error bars. The last data point includes the contribution from the electrons with energy larger than $14 \mathrm{MeV}$.

In Fig. 3(a) we plot the electron neutrino survival probabilities as a function of neutrino energy using the best fit parameters. In Fig. 3(b) we plot the recoil electron energy spectra divided by the standard prediction expected to be observed in the SuperKamiokande detector, using also the best fit parameters as in Fig. 3(a). In Fig. 3(b) we also plot the latest data from SuperKamiokande [5]. As we can see from the plot the observed data indicate some distortion mainly due to the last three data points in the higher energy bins. We, however, note from this plot that it seems difficult to exclude, at this moment, any of our predicted spectra expected from different field profiles, because of the experimental errors. We have to wait for more statistics and more careful analysis from the experimental group before drawing any definite conclusion.

In conclusion, reanalysing the RSFP mechanism as a solution to the solar neutrino problem in the light of the latest experimental data as well as the theoretical predictions, one finds that the quality of the RSFP solution to the solar neutrino anomaly crucially depends on the solar magnetic field configuration along the neutrino trajectory inside the Sun. One finds that the best fit to the observed solar neutrino data, which seems to be even better than the usual MSW solution as far as the total rates are concerned, is obtained if intensive magnetic fiels in the convective zone is assumed, in agreement with the conclusion found in ref. [30], whereas the linearly decaying magnetic field gives the worst fit. Note that the required magnitude of the free parameters involved in the process, i.e., the magnetic field strength multiplied by the neutrino magnetic moment $\mu_{\nu}\langle B\rangle$ and the squared mass difference $\Delta m^{2}$, points to the same order, $\mu_{\nu}\langle B\rangle \approx$ few times $10^{-11} \mu_{B} \cdot 10 \mathrm{kG}$ and $\Delta m^{2} \approx$ few times $10^{-8} \mathrm{eV}^{2}$, for any of the field profiles assumed in this work.

Our ignorance about the profile as well as the magnitude of the solar magnetic field makes this approach to the solar neutrino observation less predictive than its alternative approaches $[15,19,40]$. Nevertheless the presence of this mechanism opens some interesting possibilities.

One possibility is to look for any time variation of the solar neutrino signal [41] which can not be expected in other alternative solutions found in refs. [15, 19, 40]. Any time variation of the solar neutrino signal which can be attributed to some time variation of the solar magnetic field can be a good signature of this mechanism. Although SuperKamiokande has not yet confirmed any significant time variation up to experimental uncertainty this possibility remains.

Another possibility is to look for the solar $\bar{\nu}_{e}$ flux, which can not be produced in the usual MSW or vacuum oscillation case but can be produced in RSFP mechanism if the flavor mixing is included. $\bar{\nu}_{\mu}$ produced by RSFP mechanism can be converted into $\bar{\nu}_{e}$ by the usual vacuum oscillation. Ref. [42] suggests to observe (or to put upper bound of) $\bar{\nu}_{e}$ flux in the SuperKamiokande whereas ref. [43] suggests to use low 
energy solar neutrino experiment such as Borexino or Hellaz.

We finally stress that RSFP mechanism can still provide a good solution to the solar neutrino problem, comparable in quality to MSW or Just So solution, and is not excluded by the present solar neutrino data.

\section{Non-standard neutrino in- teractions}

In his seminal paper Wolfenstein [16] observed that nonstandard neutrino interactions (NSNI) with matter can also generate neutrino oscillations. In particular this mechanism could be relevant to solar neutrinos interacting with the dense solar matter along their path from the core of the sun to its surface $[44,45,46,47,48$, $49,50]$. In this case the flavor changing neutrino interactions (FCNI) are responsible for the off-diagonal elements in the neutrino propagation matrix (similar to the $\Delta m^{2} \sin ^{2} 2 \theta$ term induced by vacuum mixing). For massless neutrinos resonantly enhanced conversions can occur due to an interplay between the standard electroweak neutrino interactions and non-universal flavor diagonal neutrino interactions (FDNI) with matter $[44,51]$.

While many extensions of the standard model allow for massive neutrinos, it is important to stress that also many New Physics models predict new neutrino interactions. The minimal supersymmetric standard model without $R$-parity has been evoked as an explicit model that could provide the FCNI and FDNI needed for this mechanism. Systematic studies of the data demonstrated that resonantly enhanced oscillations induced by FCNI and FDNI for massless neutrinos [45, 49], or FCNI in combination with massive neutrinos [45, 50] can solve the solar neutrino problem.

Here we present the status of the solution to the solar neutrino problem based on NSNI follwoing the same steps of Ref. [40]. We present a comprehensive statistical analysis of this solution. Our analysis comprises both the measured total rates of Homestake [1],
GALLEX [2], SAGE [3] and SuperKamiokande [5] and the full SuperKamiokande data set (corresponding to 825 effective days of operation) including the recoil electron spectrum and the day-night asymmetry. We have not included in our $\chi^{2}$ analysis the seasonal variation but we will comment on this effect. For the solar input we take the solar neutrino fluxes and their uncertainties as predicted in the standard solar model by Bahcall and Pinsonneault (hereafter BP98 SSM) [9]. The BP98 SSM includes helium and heavy elements diffusion, as well as the new recommended value [12] for the low energy $S$-factor, $S_{17}=19_{-2}^{+4} \mathrm{eV}$ b. We also study the dependence of the allowed parameter space on the high energy ${ }^{8} \mathrm{~B}$ neutrino flux, by varying the flux normalization as a free parameter.

We find that non-standard neutrino interactions can provide a good fit to the solar neutrino data if there are rather large non-universal FDNI (of order $0.5 G_{F}$ ) and small FCNI (of order a few times $10^{-3} G_{F}$ ). Nevertheless it is shown in Ref. [40] that phenomenological contraints indicate that FCNI could only be large enough to provide $\nu_{e} \rightarrow \nu_{\tau}$ transitions, while $\nu_{e} \rightarrow \nu_{\mu}$ transitions are not relevant for the solution of the solar neutrino problem, because of strong experimental bounds. Large FDNI can only be induced by an intermediate doublet of $S U(2)_{L}$ (a scalar or a vector boson) or by a neutral vector singlet. We conclude that the minimal supersymmetric model with broken $R$-parity [55] is the favorite model for this scenario.

Any model beyond the standard electroweak theory that gives rise to the processes

$$
\begin{aligned}
& \nu_{e} f \rightarrow \nu_{\ell} f, \\
& \nu_{\alpha} f \rightarrow \nu_{\alpha} f,
\end{aligned}
$$

where (here and below) $f=u, d, e$ and $\ell=\mu, \tau$ and $\alpha=e, \mu, \tau$, is potentially relevant for neutrino oscillations in the sun, since these processes modify the effective mass of neutrinos propagating in dense matter.

The evolution equations for massless neutrinos that interact with matter via the standard weak interactions and the non-standard interactions in (3) and (4) is given by $[44,45]$ :

$$
i \frac{d}{d r}\left(\begin{array}{c}
A_{e}(r) \\
A_{\ell}(r)
\end{array}\right)=\sqrt{2} G_{F}\left(\begin{array}{cc}
n_{e}(r) & \epsilon_{\nu_{\ell}}^{f} n_{f}(r) \\
\epsilon_{\nu_{\ell}}^{f} n_{f}(r) & \epsilon_{\nu_{\ell}}^{\prime f} n_{f}(r)
\end{array}\right)\left(\begin{array}{c}
A_{e}(r) \\
A_{\ell}(r)
\end{array}\right)
$$

where $A_{e}(r)$ and $A_{\ell}(r)$ are, respectively, the probability amplitudes to detect a $\nu_{e}$ and $\nu_{\ell}$ at position $r$. For neutrinos that have been coherently produced as $\nu_{e}$ in the solar core at position $r_{0}$, the equations in (5) are subject to the boundary conditions $A_{e}\left(r_{0}\right)=1$ and $A_{\ell}\left(r_{0}\right)=0$. While $W$-exchange of $\nu_{e}$ with the background electrons gives rise to the well known forward scattering am- 
plitude $\sqrt{2} G_{F} n_{e}(r)$, the FCNI in (3) induce a flavor changing forward scattering amplitude $\sqrt{2} G_{F} \epsilon_{\nu_{\ell}}^{f} n_{f}(r)$ and the non-universal FDNI are responsible for the flavor diagonal entry $\sqrt{2} G_{F} \epsilon^{\prime}{ }_{\nu_{\ell}}^{f} n_{f}(r)$ in eq. (5). Here

$$
n_{f}(r)= \begin{cases}n_{n}(r)+2 n_{p}(r) & f=u \\ 2 n_{n}(r)+n_{p}(r) & f=d\end{cases}
$$

is the respective fermion number density at position $r$ in terms of the proton [neutron] number density $n_{p}(r)$ $\left[n_{n}(r)\right]$ and

$$
\varepsilon=\epsilon_{\nu_{\ell}}^{f} \equiv \frac{G_{\nu_{e} \nu_{\ell}}^{f}}{G_{F}}, \varepsilon^{\prime}=\epsilon_{\nu_{\ell}}^{\prime f} \equiv \frac{G_{\nu_{\ell} \nu_{\ell}}^{f}-G_{\nu_{e} \nu_{e}}^{f}}{G_{F}}
$$

describe, respectively, the relative strength of the FCNI in (3), and the new flavor diagonal, but non-universal interactions in (4). $G_{\nu_{\alpha} \nu_{\beta}}^{f}(\alpha, \beta=e, \mu, \tau)$ denotes the effective coupling of the four-fermion operator

$$
\mathcal{O}_{\nu}^{f} \equiv\left(\overline{\nu_{\alpha}} \nu_{\beta}\right)(\bar{f} f)
$$

that gives rise to such interactions. The Lorentz structure of $\mathcal{O}_{\nu}^{f}$ depends on the New Physics that induces this operator. Operators which involve only left-handed neutrinos (and which conserve total lepton number $L$ ) can be decomposed into a $(V-A) \otimes(V-A)$ and a $(V-A) \otimes(V+A)$ component. (Any single New Physics contribution that is induced by chiral interactions yields only one of these two components.) It is, however, important to note that only the vector part of the background fermion current affects the neutrino propagation for an unpolarized medium at rest [16, 52]. Hence only the $(V-A) \otimes(V)$ part of $\mathcal{O}_{\nu}^{f}$ is relevant for neutrino oscillations in normal matter. One mechanism to induce such operators is due to the exchange of heavy bosons that appear in various extensions of the standard model. An alternative mechanism arises when extending the fermionic sector of the standard model and is due to $Z$-induced flavor-changing neutral currents (FCNCs). For a discussion of $Z$-induced FCNC effects on solar neutrinos, see Refs. [53, 54].

A resonance occurs when the diagonal entries of the evolution matrix in eq. (5) coincide at some point $r_{r e s}$ along the trajectory of the neutrino, leading to the resonance condition

$$
\epsilon_{\nu_{\ell}}^{f} n_{f}\left(r_{r e s}\right)=n_{e}\left(r_{r e s}\right)
$$

An immediate consequence is that new FDNI for $f=e$ alone cannot induce resonant neutrino flavor conversions.

As it was observed in Ref. [40] that only $\nu_{e} \rightarrow$ $\nu_{\tau}$ conversions are compatible with the existing phenomenological constraints on $\epsilon_{\nu_{\ell}}^{f}$ and $\epsilon_{\nu_{\ell}}^{\prime f}$. We note that in the minimal supersymmetric standard model with broken $R$-parity [55] the relevant parameters are given by

$$
\epsilon_{\nu_{\tau}}^{d}=\frac{\lambda_{331}^{\prime}{ }^{*} \cdot \lambda_{131}^{\prime}}{4 M_{\tilde{b}}^{2} \sqrt{2} G_{F}}, \epsilon_{\nu_{\tau}}^{\prime d}=\frac{\left|\lambda_{331}^{\prime}\right|^{2}-\left|\lambda_{131}^{\prime}\right|^{2}}{4 M_{\tilde{b}}^{2} \sqrt{2} G_{F}},
$$

in terms of the trilinear couplings $\lambda_{i j k}^{\prime}$ and the bottom squark mass $M_{\tilde{b}}$.

The neutrino evolution matrix in eq. (5) vanishes in vacuum and is negligibly small for the matter densities of the earth's atmosphere. Therefore the probability of finding an electron neutrino arriving at the detector during day time is easily obtained by evolving the equations in (5) from the neutrino production point to the solar surface. Furthermore, typically there are many oscillations between the neutrino production and detection point and a resonance. Therefore the phase information before and after the resonance is usually lost after integration over the production and detection region and one may use classical survival probabilities. Then at day time we have [45]

$$
P_{\nu_{e} \rightarrow \nu_{e}}^{\text {day }}=\left|A_{e}\left(r_{s}\right)\right|^{2} \simeq \frac{1}{2}+\left(\frac{1}{2}-P_{c}\right) \cos 2 \theta_{m}^{p} \cos 2 \theta_{m}^{s},
$$

where $r_{s}$ is the solar surface position and in the analytic expression in eq. (11) we denote by $\theta_{m}^{p}$ and $\theta_{m}^{s}$, respectively, the effective, matter-induced mixing at the neutrino production point and at the solar surface. In terms of the New Physics parameters $\varepsilon, \varepsilon^{\prime}$ and the fermion densities the effective mixing is given by $[44,45]$

$$
\tan 2 \theta_{m}=\frac{2 \epsilon_{\nu_{\ell}}^{f} n_{f}}{\epsilon_{\nu_{\ell}}^{\prime f} n_{f}-n_{e}} .
$$

Note that $\tan 2 \theta_{m}=2 \epsilon_{\nu_{\ell}}^{e} /\left(\epsilon_{\nu_{\ell}}^{\prime e}-1\right)$ is constant for $f=e . \quad P_{c}$ is the level crossing probability. The approximate Landau-Zener expression is $[44,45]$

$$
P_{c}=\exp [-\pi \gamma / 2]
$$

with

$$
\gamma=4 \sqrt{2} G_{F}\left|\frac{\left(\epsilon_{\nu_{\ell}}^{f} / \epsilon_{\nu_{\ell}}^{\prime f}\right)^{2}}{\epsilon_{\nu_{\ell}}^{\prime f}} \cdot \frac{n_{e}}{\frac{d}{d x}\left(\frac{n_{f}}{n_{e}}\right)}\right|_{\text {res }} .
$$

When neutrinos arrive at the detector during the night, a modification of the survival probability has to be introduced since the non-standard neutrino interactions with the terrestrial matter may regenerate electron neutrinos that have been transformed in the sun. Assuming that the neutrinos reach the Earth as an incoherent mixture of the effective mass-eigenstates $\nu_{1}$ and $\nu_{2}$ the 
survival probability during night-time can be written as $[56]$ :

$$
P_{\nu_{e} \rightarrow \nu_{e}}^{\text {night }}=\frac{P_{\nu_{e} \rightarrow \nu_{e}}^{\text {day }}-\sin ^{2} \theta_{m}^{s}+P_{2 e}\left(1-2 P_{\nu_{e} \rightarrow \nu_{e}}^{\text {day }}\right)}{\cos 2 \theta_{m}^{s}}
$$

Here $P_{2 e}$ is the probability of a transition from the state $\nu_{2}$ to the flavor eigenstate $\nu_{e}$ along the neutrino path in the Earth.

For our analysis we assume a step function profile for the Earth matter density, which has been shown to be a good approximation in other contexts (see e.g. Ref. [57] for a recent analysis of matter effects for atmospheric neutrinos). Then the earth matter effects on the neutrino propagation correspond to a parametric resonance and can be calculated analytically [25],

$$
P_{2 e}=\sin ^{2} \theta_{m}^{s}+W_{1}^{2} \cos 2 \theta_{m}^{s}+W_{1} W_{3} \sin 2 \theta_{m}^{s},
$$

where the parameters $W_{1}$ and $W_{3}$ contain all the information of the Earth density and are defined in Ref. [25]. (The only difference is that in our case also the offdiagonal element of the neutrino evolution matrix varies when the neutrino propagates through the earth matter.)

It is this interaction with the terrestrial matter that can produce a day-night variation of the solar neutrino flux and, consequently, a seasonal modulation of the data. (Note that this seasonal variation is of a different nature than the one expected for vacuum oscillations from the change of the baseline due to the eccentricity of the earth's orbit around the sun.)

Our main goal is to determine the values of $\varepsilon$ and $\varepsilon^{\prime}$ that can explain the experimental observations without modifying the standard solar model predictions.

First we consider the data on the total event rate measured by the Chlorine $(\mathrm{Cl})$ experiment [1], the Gallium (Ga) detectors GALLEX [2] and SAGE [3] and the water Cherenkov experiment SuperKamiokande (SK) [5]. We compute the allowed regions in parameter space according to the BP98 SSM [9] and compare the results with the regions obtained for an arbitrary normalization $f_{B}$ of the high energy neutrino ${ }^{8} \mathrm{~B}$ neutrino fluxes.

We use the minimal $\chi^{2}$ statistical treatment of the data following the analyses of Refs. [59, 34].
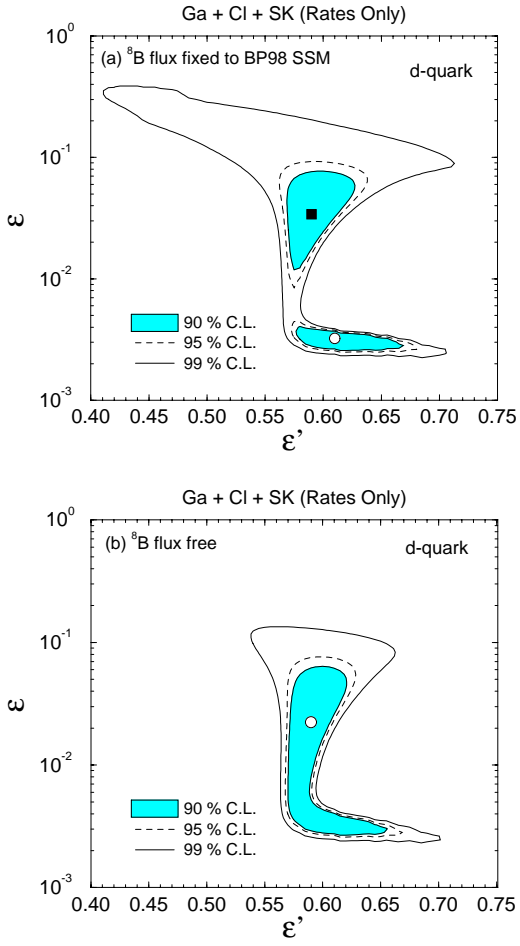

Figure 4. Region of $\varepsilon=\epsilon_{\nu}^{d}$ and $\varepsilon^{\prime}=\epsilon^{\prime d}{ }_{\nu}^{d}$ which can explain the total rates measured by the Homestake, GALLEX, SAGE and SuperKamiokande solar neutrino experiments in terms of non-standard neutrino interactions with $d$-quarks. (a) The best fit (indicated by the open circle) is obtained for $\left(\varepsilon, \varepsilon^{\prime}\right)=(0.0032,0.610)$ with $\chi_{\min }^{2}=2.44$ for $4-2=2$ DOF. A second (local) $\chi^{2}$ minimum (indicated by the solid square $)$ is found at $\left(\varepsilon, \varepsilon^{\prime}\right)=(0.034,0.610)$ with $\chi^{2}=2.63$. (b) Allowing for an arbitrary ${ }^{8} \mathrm{~B}$ flux normalization $f_{B}$, the best fit (indicated by the open circle) is obtained for $\left(\varepsilon, \varepsilon^{\prime}\right)=(0.022,0.590)$ and $f_{B}=1.36$ with $\chi_{\text {min }}^{2}=0.91$ for $4-3=1$ DOF.

In Fig. 4 the allowed regions in the parameter space of $\epsilon_{\nu}^{d}$ and $\epsilon_{\nu}^{\prime}{ }_{\nu}^{d}$ for neutrino scattering off $d$-quarks are shown at 90, 95 and $99 \%$ confidence level (CL). In Fig. 4a, the ${ }^{8} \mathrm{~B}$ flux is fixed by the BP98 SSM prediction $\left(f_{B}=1\right)$. The best fit point of this analysis is found at

$$
\epsilon_{\nu}^{d}=3.2 \times 10^{-3} \quad \text { and } \quad \epsilon_{\nu}^{\prime d}=0.61
$$

with $\chi_{\min }^{2}=2.44$ for $4-2=2$ degrees of freedom (DOF). Allowing an arbitrary ${ }^{8} \mathrm{~B}$ flux normalization, a different best fit point is obtained for $\left(\epsilon_{\nu}^{d}, \epsilon_{\nu}^{\prime d}\right)=$ $\left(2.2 \times 10^{-2}, 0.59\right)$ and $f_{B}=1.36$ with $\chi_{\text {min }}^{2}=0.91$ for $4-3=1 \mathrm{DOF}$. The result of this analysis is shown in Fig. 4b. (Effects due to deviations of the hep neutrino flux from the standard solar model prediction are expected to be less significant and we do not consider them in this work.) 

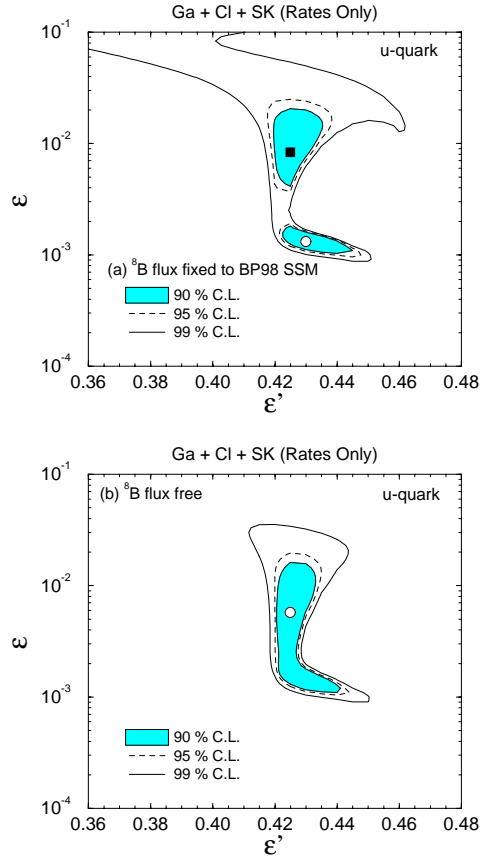

Figure 5. Same as in Fig. 4 but for $u$-quarks. (a) The best fit (indicated by the open circle) is obtained for $\left(\varepsilon, \varepsilon^{\prime}\right)=(0.0013,0.430)$ with $\chi_{\min }^{2}=2.75$ for $4-2=2$ DOF. A second (local) $\chi^{2}$ minimum (indicated by the solid square $)$ is found at $\left(\varepsilon, \varepsilon^{\prime}\right)=(0.0083,0.425)$ with $\chi^{2}=2.70$. (b) Allowing for an arbitrary ${ }^{8} \mathrm{~B}$ flux normalization $f_{B}$, the best fit (indicated by the open circle) is obtained for $\left(\varepsilon, \varepsilon^{\prime}\right)=(0.0058,0.425)$ and $f_{B}=1.34$ with $\chi_{\text {min }}^{2}=0.96$ for $4-3=1$ DOF.

In Fig. 5 the allowed regions in the parameter space of $\epsilon_{\nu}^{u}$ and $\epsilon_{\nu}^{\prime u}$ for neutrino scattering off $u$-quarks are shown at 90,95 and $99 \%$ CL. In Fig. 5a, the ${ }^{8}$ B flux is fixed by the BP98 SSM prediction. The best fit point of this analysis is found at

$$
\epsilon_{\nu}^{u}=1.32 \times 10^{-3} \quad \text { and } \quad \epsilon^{\prime \prime}{ }_{\nu}^{u}=0.43,
$$

with $\chi_{\min }^{2}=2.64$ for two DOF. Allowing an arbitrary ${ }^{8} \mathrm{~B}$ flux normalization $f_{B}$, a different best fit point is obtained for $\left(\epsilon_{\nu}^{u}, \epsilon^{\prime \prime}\right)=\left(5.8 \times 10^{-3}, 0.425\right)$ and $f_{B}=1.34$ with $\chi_{\min }^{2}=0.96$ for one DOF. The result of this analysis is shown in Fig. $5 \mathrm{~b}$.

It is remarkable that the neutrino flavor conversion mechanism based on NSNI provides quite a good fit to the total rates despite the fact that the conversion probabilities (11) and (15) do not depend on the neutrino energy. This is unlike the case of the vacuum and the MSW conversion mechanisms which provide the appropriate energy dependence to yield a good fit. For NSNI the only way to distinguish between neutrinos of different energies is via the position of the resonance $r_{\text {res }}$. Note that according to eq. (9), $r_{\text {res }}$ is a function of $\varepsilon^{\prime}$ only. In the Sun, $n_{e} / n_{f}(f=d, u)$ is a smooth and monotonic function of the distance from the solar center $r$, allowing to uniquely determine $r_{\text {res }}$ for a given value of $\varepsilon^{\prime}$. Consequently, it follows that a resonance can only occur if $\epsilon_{d}^{\prime} \in[0.50,0.77]$ for NSNI with $d$-quarks or $\epsilon_{u}^{\prime} \in[0.40,0.46]$ for NSNI with $u$-quarks. For both cases the major part of these intervals corresponds to $r_{\text {res }} \lesssim 0.2 R_{\odot}\left(R_{\odot}\right.$ being the solar radius). For $\epsilon_{d, u}^{\prime}$ within the $90 \%$ CL regions (indicated in Fig. 4 and Fig. 5) we find $r_{r e s} \approx 0.1 R_{\odot}$. Since the nuclear reactions that produce neutrinos with higher energies in general take place closer to the solar center (see chapter 6 of Ref. [11] for the various spatial distributions of the neutrino production reactions), a resonance position close to the solar center implies that predominantly the high energy neutrinos are converted by a resonant transition. For $r_{r e s} \approx 0.1 R_{\odot}$ practically all ${ }^{8} \mathrm{~B}$-neutrinos cross the resonance layer, fewer ${ }^{7} \mathrm{Be}-$ neutrinos pass through the resonance, while most of the $p p$-neutrinos are not be affected by the resonance since their production region extends well beyond the resonance layer. Therefore for most of the allowed region in Fig. 4 and Fig. 5 the corresponding average survival probability fulfill the following relations:

$$
\left\langle P\left({ }^{8} \mathrm{~B}\right)\right\rangle\left\langle\left\langle P\left({ }^{7} \mathrm{Be}\right)\right\rangle\langle\langle P(p p)\rangle .\right.
$$

We note that the above relation is still valid when taking into account that a significant fraction of the $p p$ neutrinos crosses the resonance layer twice, if they are produced just outside resonance. This is - roughly speaking - because a $\nu_{e}$ which undergoes a resonant flavor transition when entering the solar interior at $r_{\text {res }}$ is reconverted into a $\nu_{e}$ at the second resonance when it emerges again from the solar core. In our numerical calculations we properly take into account the effects of such double resonances.

An immediate consequence of the relation in eq. (19) is that as long as $f_{B}=1$ the NSNI solution predicts that $R_{S K}<R_{C l}<R_{G a}$, which is inconsistent with the observed hierarchy of the rates, $R_{C l}<R_{S K}<R_{G a}$, leading to a somewhat worse fit than the standard MSW solutions. However when treating $f_{B}$ as a free parameter, for $f_{B} \sim 1.3-1.4$ the SK rate is sufficiently enhanced to give the correct relation between the rates. In this case also the neutral current contribution from $\nu_{\mu, \tau} e^{-}$scattering is increased due to a larger $\nu_{\mu, \tau}$ flux, which is consistent with the Super-Kamiokande observations. We find that for the best fit points for $\left(\varepsilon, \varepsilon^{\prime}\right)$ in Figs. $4 \mathrm{~b}$ and $5 \mathrm{~b}$ and $f_{B} \sim 1.35$ the survival probability for ${ }^{8} \mathrm{~B},{ }^{7} \mathrm{Be}$ and $p p$-neutrinos are $\sim 0.24,0.4$ and 0.7 , respectively.

Next, we consider the zenith angle dependence of the solar neutrino data of the SuperKamiokande experiment. As mentioned above, NSNI with matter may affect the neutrino propagation through the earth resulting in a difference between the event rates during day and night time. The data obtained by the SuperKamiokande collaboration are divided into five bins containing the events observed at night and one bin for the events collected during the day [60] and have been 
averaged over the period of SuperKamiokande operation: 403.2 effective days for the day events and 421.5 effective days for the night events. The experimental results suggest an asymmetry between the total data collected during the day $(D)$ and the total data observed during the night $(N)[60]$ :

$$
A=2 \frac{N-D}{N+D}=0.065 \pm 0.031 \text { (stat.) } \pm 0.013 \text { (syst.) . }
$$

In order to take into account the earth matter effect we define the a $\chi^{2}$-function that characterizes the deviations of the six measured $\left(Z_{i}^{\text {obs }}\right)$ from the predicted $\left(Z_{i}^{t h}\right)$ values of the rate as a function of zenith angle (see Ref. [40]).
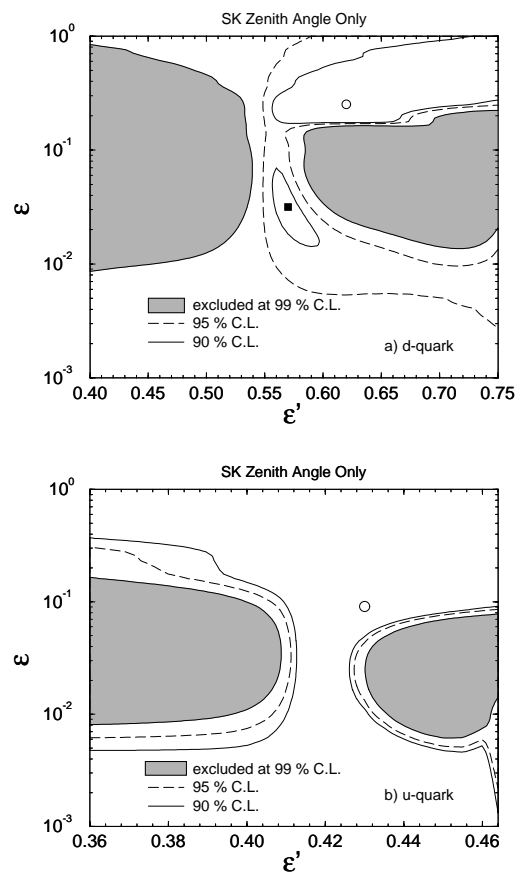

Figure 6. Region of $\varepsilon=\epsilon_{\nu}^{d}$ and $\varepsilon^{\prime}=\epsilon^{\prime d}{ }_{\nu}$ which is excluded by day and night data (contained in $1+5$ bins) as measured by the SuperKamiokande solar neutrino experiment in terms of non-standard neutrino interactions with (a) $d$-quarks and (b) $u$-quarks. For $d$-quarks, the best fit (indicated by the open circle) is obtained for $\left(\varepsilon, \varepsilon^{\prime}\right)=(0.251,0.620)$ and $\alpha_{Z}=0.819$ with $\chi_{\min }^{2}=1.10$ for $6-3=3$ DOF. A second (local) $\chi^{2}$ minimum (indicated by the solid squared) is found at $\left(\varepsilon, \varepsilon^{\prime}\right)=(0.0316,0.570)$ and $\alpha_{Z}=1.02$ with $\chi^{2}=5.20$. For $u$-quarks, the best fit (indicated by the open circle) is obtained for $\left(\varepsilon, \varepsilon^{\prime}\right)=(0.229,0.690)$ and $\alpha_{Z}=0.685$ with $\chi_{\min }^{2}=1.44$ for $6-3=3 \mathrm{DOF}$.

In Fig. 6 we show the allowed regions in the $\left(\varepsilon, \varepsilon^{\prime}\right)$ parameter space for neutrino scattering off $d$ - and $u$ quarks, respectively. The contours in Fig. 6 correspond to the allowed regions at 90, 95 and $99 \%$ CL. The best fit (indicated by the open circle) is obtained for $\left(\epsilon_{\nu}^{d}, \epsilon^{\prime \prime}{ }_{\nu}^{d}\right)=(0.251,0.62)$ and $\alpha_{Z}=0.819$ with $\chi_{\min }^{2}=1.10$ for neutrino scattering off $d$-quarks and at $\left(\epsilon_{\nu}^{u}, \epsilon^{\prime \prime}{ }_{\nu}\right)=(0.229,0.690)$ and $\alpha_{Z}=0.685$ with $\chi_{\min }^{2}=1.44$ for neutrino scattering off $u$-quarks (having $6-3=3$ DOF in both cases).
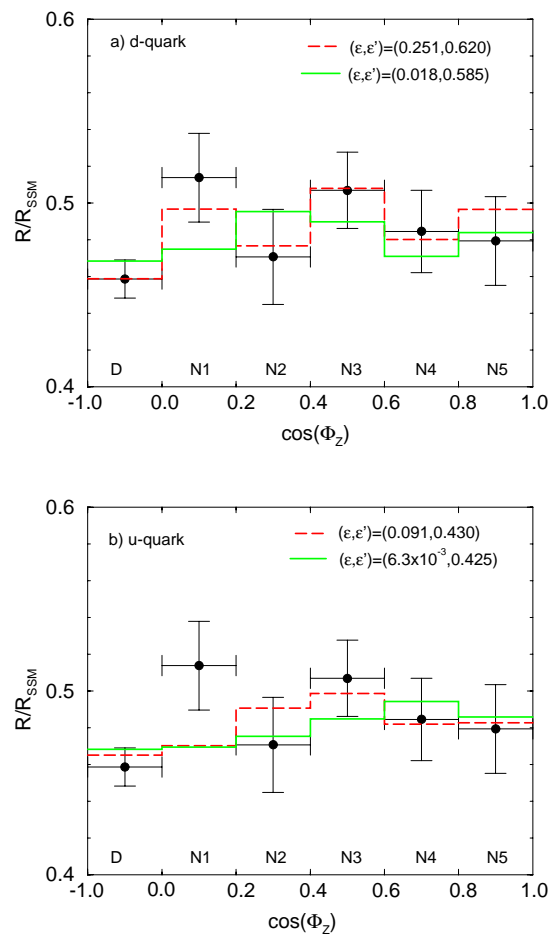

Figure 7. Expected zenith angle dependence with the our best fit values of $\left(\varepsilon, \varepsilon^{\prime}\right)$ determined by the SK Zenith angle only as well as the combined analysis for (a) $d$-quarks and (b) $u$-quarks.

Finally, in Fig. 7 we show the expected zenith angle distributions for SuperKamiokande using the values of $\left(\epsilon, \epsilon^{\prime}\right)$ determined by the best fit. For comparison, we also present in this figure the expected zenith angle distributions for the best fit values of $\left(\epsilon, \epsilon^{\prime}\right)$ found in the combined analysis (that will be discussed later).

We also consider the measurements of the recoil electron spectrum by SuperKamiokande [60]. Data are divided into 18 bins. 17 of these bins have a width of $0.5 \mathrm{MeV}$ and are grouped into two bins for a super low energy analysis with energies between $5.5 \mathrm{MeV}$ and 6.5 $\mathrm{MeV}$ and 15 bins with energies ranging from $6.5 \mathrm{MeV}$ (the low energy limit) to $14 \mathrm{MeV}$. The last bin includes all the events with energies larger than $14 \mathrm{MeV}$.

Since the electron neutrino survival probability does not depend on the neutrino energy in the NSNI scenario, the spectral distortion of the recoil electrons from ${ }^{8} \mathrm{~B}$ neutrino due to the presence of a $\nu_{\mu, \tau}$ component in the neutrino flux is expected to be very small [61] and therefore, even a relatively small spectral distortion (such as the one expected in small mixing angle MSW solution) could rule out this solution.

The $\chi^{2}$-function that characterizes the deviations of the measured $\left(S_{i}^{o b s}\right)$ from the predicted $\left(S_{i}^{t h}\right)$ values for the electron recoil spectrum therefore provides an important test of the NSNI solution [40] Fitting the 
present data to our scenario we obtain $\chi_{\min }^{2}=20.0$ for $18-1=17$ DOF, which is still acceptable at the $27 \% \mathrm{CL}$

The earth matter effects on neutrino flavor transitions induce a seasonal variation of the data (beyond the expected variation of the solar neutrino flux due to the eccentricity of the earth's orbit) due to the variation of the day and night time during the year. Since these variations can be relevant to other neutrino oscillation scenarios [62], a positive signal could help to distinguish the various solutions and it is worthwhile to analyze the effects of such a variation in the NSNI scenario.
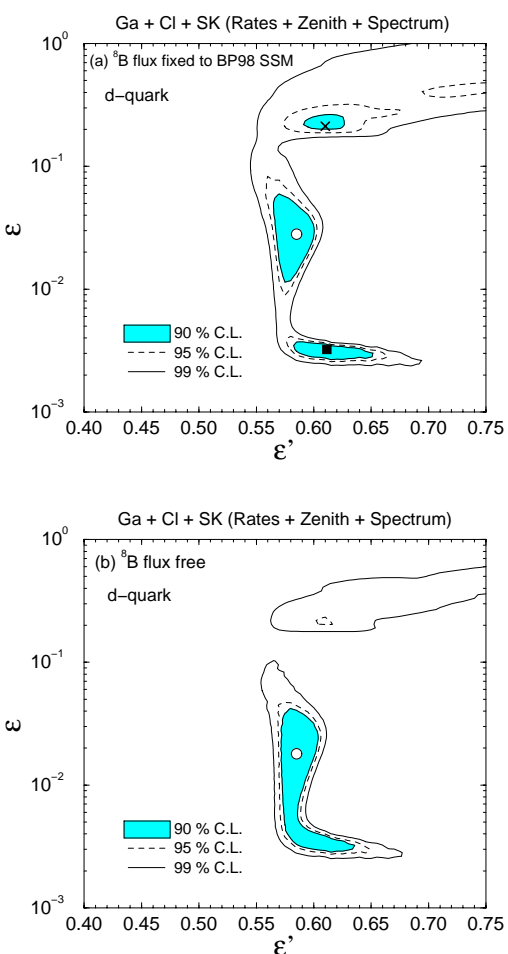

Figure 8. The allowed region for $\varepsilon=\epsilon_{\nu}^{d}$ and $\varepsilon^{\prime}=\epsilon^{\prime d}{ }_{\nu}$ obtained by the combined analysis using 4 rates +6 zenith angle bins +18 spectrum bins for non-standard neutrino interactions with $d$-quarks. (a) Fixing $f_{B}=1$ the best fit (indicated by the open circle) is obtained for $\left(\varepsilon, \varepsilon^{\prime}\right)=$ $(0.028,0.585)$ with $\chi_{\min }^{2}=29.05$ for $28-4=24$ DOF. There are two additional (local) $\chi^{2}$ minima at $\left(\varepsilon, \varepsilon^{\prime}\right)=$ $(0.0033,0.610)$ with $\chi^{2}=29.40$ (indicated by the solid square) and $\left(\varepsilon, \varepsilon^{\prime}\right)=(0.21,0.61)$ with $\chi^{2}=33.1$ (indicated by the cross). (b) Same as in (a) but allowing a free $f_{B}$. The best fit (indicated by the open circle) is obtained for $\left(\varepsilon, \varepsilon^{\prime}\right)=(0.018,0.585)$ and $f_{B}=1.38$ with $\chi_{\min }^{2}=26.62$ for $28-5=23 \mathrm{DOF}$

The present SK solar neutrino data do not provide any conclusive evidence in favor of such a variation. It only indicates that the variation might be larger for recoil electron energies above $11.5 \mathrm{MeV}$. In our scenario, however, we do not expect any correlation between the seasonal variation and the recoil electron energies, since the electron neutrino survival probability does not depend on the neutrino energy. Therefore any range of parameters that leads to a considerable seasonal modulation for energies above $11.5 \mathrm{MeV}$ is disfavored by the data for lower energies. However, for the range of parameters $\left(\varepsilon, \varepsilon^{\prime}\right)$ that can solve the solar neutrino problem, earth regeneration effects are never strong enough to induce a significant seasonal variation. Hence taking into account the data on seasonal variations neither changes the shape of the allowed region, nor the best fit points.
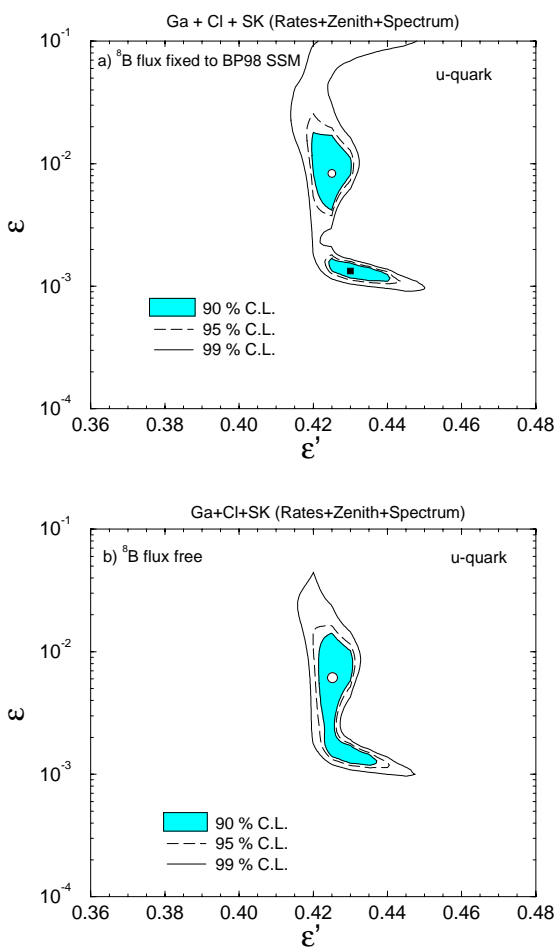

Figure 9. Same as in Fig. ?? but for $u$-quarks. (a) Fixing $f_{B}=1$ the best fit (indicated by the open circle) is obtained for $\left(\varepsilon, \varepsilon^{\prime}\right)=(0.0083,0.425)$ with $\chi_{\min }^{2}=28.45$ for $28-4=24$ DOF. A second (local) $\chi^{2}$ minimum is found at $\left(\varepsilon, \varepsilon^{\prime}\right)=(0.0013,0.430)$ with $\chi^{2}=30.27$ (indicated by the solid square) (b) Same as in (a) but allowing a free $f_{B}$. The best fit (indicated by the open circle) is obtained for $\left(\varepsilon, \varepsilon^{\prime}\right)=(0.0063,0.426)$ and $f_{B}=1.34$ with $\chi_{\min }^{2}=26.59$ for $28-5=23$ DOF.

Our final result is the fit derived from the combined analysis of all presently available solar neutrino data. In Fig. 8 and Fig. 9 we show the allowed regions for $\left(\epsilon_{\nu}^{d}, \epsilon^{\prime}{ }_{\nu}^{d}\right)$ and $\left(\epsilon_{\nu}^{u}, \epsilon^{\prime}{ }_{\nu}^{u}\right)$, respectively, using both the results from the total rates from the Chlorine, GALLEX, SAGE and SuperKamiokande solar neutrino experiments together with the 6 bins from the $\mathrm{Su}-$ perKamiokande zenith angle data discussed previously. Although adding the spectral information to our analysis does not change the shape of allowed regions nor the best fit points, it is included in order to determine the quality of the global fit. However, we do not take into account the seasonal variation in our combined $\chi^{2}$ analysis, since the effect is negligible. 
For neutrino scattering off $d$-quarks the best fit for the combined data is obtained for

$$
\epsilon_{\nu}^{d}=0.028 \quad \text { and } \quad \epsilon_{\nu}^{\prime d}=0.585
$$

with $\chi_{\min }^{2}=29.05$ for $28-4=24 \mathrm{DOF}$, corresponding to a solution at the $22 \% \mathrm{CL}$ (see Fig. 8a). Allowing $f_{B} \neq 1$, the best fit is found at $\left(\epsilon_{\nu}^{d}, \epsilon^{\prime}{ }_{\nu}^{d}\right)=(0.018,0.585)$ and $f_{B}=1.38$ with $\chi_{\text {min }}^{2}=26.62$ for $28-5=23$ DOF, corresponding to a solution at the $27 \%$ CL (see Fig. 8b). For neutrino scattering off $u$-quarks the best fit for the combined data is obtained for

$$
\epsilon_{\nu}^{u}=0.0083 \quad \text { and } \quad \epsilon^{\prime \prime}{ }_{\nu}=0.425
$$

with $\chi_{\min }^{2}=28.45$ for $28-4=24$ DOF corresponding to a solution at the $24 \%$ CL (see Fig. 9a). Allowing $f_{B} \neq 1$, the best fit is obtained for $\left(\epsilon_{\nu}^{u}, \epsilon^{\prime}{ }_{\nu}\right)=$ $(0.0063,0.426)$ and $f_{B}=1.34$ with $\chi_{\text {min }}^{2}=26.59$ for $28-5=23$ DOF, corresponding to a solution at the $27 \%$ CL (see Fig. 9b). These results have to be compared with the fit for standard model neutrinos, that do not oscillate (where the CL is smaller than $10^{-7}$ ), as well as to the standard solutions of the solar neutrino problem in terms of usual neutrino oscillations (36\% CL) $[18,19]$.

Finally, in Fig. 7 we show the expected zenith angle distributions for SuperKamiokande using the best fitted values of $\left(\epsilon, \epsilon^{\prime}\right)$ from the combined analysis.

According to our $\chi^{2}$ analysis non-standard neutrino interactions (NSNI) can provide a good fit to the solar neutrino data provided that there are rather large non-universal FDNI (of order $0.5 G_{F}$ ) and small FCNI (of order $10^{-2}-10^{-3} G_{F}$ ). The fit to the observed total rate, day-night asymmetry, seasonal variation and spectrum distortion of the recoil electron spectrum is comparable in quality to the one for standard neutrino oscillations.

From the model-independent analysis of Ref. [40] we leran that NSNI induced by the exchange of heavy bosons cannot provide large enough $\nu_{e} \rightarrow \nu_{\mu}$ transitions, while $\nu_{e}-\nu_{\tau}$ FCNI in principle could be sufficiently strong. However, the current bounds will be improved by the up-coming $B$-factories, providing an independent test of the NSNI solution. The required large non-universal FDNI (for $\nu_{e}$ transitions into both $\nu_{\mu}$ and $\nu_{\tau}$ ) can be ruled out by the upper bounds on lepton universality, unless they are induced by an intermediate doublet of $S U(2)_{L}$ (a scalar or a vector boson) or by a neutral vector singlet. For $\nu_{e} \rightarrow \nu_{\mu}$ there exists a bound due to the limit on compositeness in this case, but for $\nu_{e} \rightarrow \nu_{\tau}$ there is no significant constraint at present.

Generically only very few models can fulfill the requirements needed for the solution discussed in this paper: massless neutrinos, small FCNI and relatively large non-universal FDNI. As for the vector bosons the most attractive scenario is to evoke an additional $U(1)_{B-3 L_{\tau}}$ gauge symmetry (where $B$ is the baryon number and $L_{\tau}$ denotes the tau lepton number), which would introduce an additional vector singlet that only couples to the third generation leptons and quarks [63]. Among the attractive theories beyond the standard model where neutrinos are naturally massless as a result of a protecting symmetry, are supersymmetric $S U(5)$ models [64] that conserve $B-L$, and theories with an extended gauge structure such as $S U(3)_{C} \otimes S U(3)_{L} \otimes$ $U(1)_{N}$ models [65], where a chiral symmetry prevents the neutrino from getting a mass. These particular models, however, do not contribute significantly to the specific interactions we are interested in this paper. $S U(5)$ models have negligible NSNI since they are mediated by vector bosons which have masses at the GUT scale. $S U(3)_{C} \otimes S U(3)_{L} \otimes U(1)_{N}$ models can provide large $\epsilon_{e}$ and $\epsilon_{e}^{\prime}$, but these models do not induce NSNI with quarks. From eq. (9) it follows that no resonant conversion can occur in this case.

Therefore we conclude that the best candidate for the scenario we studied are supersymmetric models with broken $R$-parity, where the relevant NSNI are mediated by a scalar doublet, namely the "left-handed" bottom squark. Although in this model neutrino masses are not naturally protected from acquiring a mass, one may either evoke an additional symmetry or assume that non-zero neutrino masses are not in a range that would spoil the solution in terms of the nonstandard neutrino oscillations.

\section{Violation of the equivalence principle}

Let me brefly mention that a different solution to the solar neutrino anomaly based on the violation of the equivalence principle was recently revisited [72]. In this context, neutrino mixing and flavor oscillations are induced by gravitational forces. It is assumed that neutrinos of diferent species will incur different time delay to the weak, static gravitational filed in the intervening space on their way from the sun to the earth. Their motion in this gravitational field is described assuming a different neutrino gravitational coupling for each neutrino type. In this way, weak intereacting eigenstates and gravitational interacting eigenstates will be related by a unitary transformation that can be parametrized, assuming only two neutrino flavors, by a single parameter, the mixing angle $\theta_{G}$ which can lead to neutrino oscillations. The evolution equations for these flavors, which are assumed to be degenerate in mass, propagating through the gravitational potential $\phi(r)$ in absence of matter is [73]: 


$$
i \frac{d}{d t}\left(\begin{array}{c}
\nu_{e} \\
\nu_{x}
\end{array}\right)=2 E \phi(r) \Delta \gamma\left(\begin{array}{cc}
\cos 2 \theta_{G} & \sin 2 \theta_{G} \\
\sin 2 \theta_{G} & -\cos 2 \theta_{G}
\end{array}\right)\left(\begin{array}{c}
\nu_{e} \\
\nu_{x}
\end{array}\right)
$$

where $E$ is the neutrino energy, $\nu_{x}=\nu_{\mu}, \nu_{\tau}$ or $\nu_{S} ; \Delta \gamma$ is the quantity which measures the magnitude of the violation of the equivalence principle and it is the difference of the gravitational couplings between the two neutrinos involved normalized by the sum.

\begin{tabular}{ccccc}
\hline \hline Case & $\sin ^{2} 2 \theta_{G}$ & $|\phi \Delta \gamma|$ & $f_{B}$ & $\chi_{\text {min }}^{2}$ \\
\hline Rates & 1.0 & 1.71 & fix $=1$ & 1.49 \\
Rates & 1.0 & 1.70 & 0.81 & 0.32 \\
Spectrum & 0.98 & 1.00 & 0.80 & 15.8 \\
Combined & 1.0 & 1.65 & 0.82 & 22.0 \\
\hline
\end{tabular}

TABLE II. The best fitted parameters and $\chi_{\min }^{2}$ for the violation of the equivalence principle scenario.

Applying the same statistical treatment shown in Ref. [34], the allowed region in the parameter space $|\phi \Delta \gamma| \times \sin ^{2} 2 \theta_{G}$ determined only by the rates is shown in Fig. 10 (a), which was taken directly from Ref. [72]. It was found that, considering a free normalization factor $f_{B}$ for the ${ }^{8} B$ neutrino flux, $\xi_{\text {mim }}^{2}=0.32$ for $3-3$ degrees of
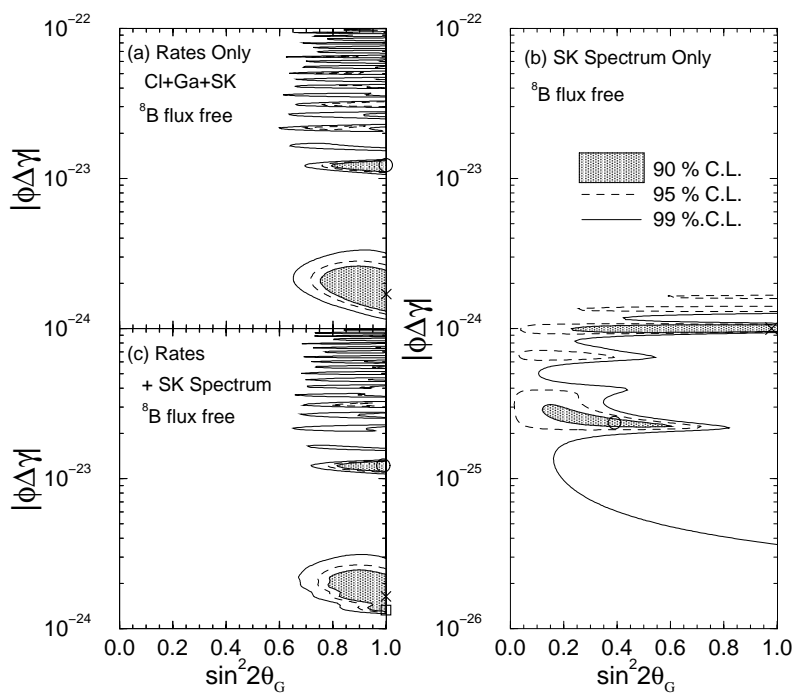

Figure 10. Allowed parameter region for (a) the rates, (b) SuperKamiokande spectrum, and (c) rates plus Superkamiokande spectrum combined. The best fit points are indicated by the crosses and the local best fit points in other $90 \%$ C.L. islands are indicated by the open circles.

freedom. Considering a spectral shape analysis, $\xi_{\text {mim }}^{2}=$ 15.8 for $18-3$ degrees of freedom which result is shown in
Fig. 10(b). Finally, the combined result (rates +spectrum) is shown in Fig. 10(c), which provides $\xi_{\text {mim }}^{2}=$ 22.0 for 21-3 degrees of freedom. Details of this picture and the values of the best fit points are found in Table II.

In conclusion, a solution to the solar neutrino anomaly which is coomparable in quality of the fit to the other suggested ones, can be found by means of the violation of the equivalence principle.

\section{Conclusion}

Even though the conventional oscillation mechanisms can be considered the most plausible solutions to the solar neutrino problem, it is important to realize that in general New Physics in the neutrino sector include neutrino masses and mixing, as well as new neutrino interactions which can generate, in principle, large neutrino magnetic moment, neutrino flavor-changing and nonuniversal processes, violation of the equivalence principle. While it is difficult to explain the atmospheric neutrino problem [74] and the LSND anomalies [75] by these alternative mechanisms, we have shown in this review that a solution of the solar neutrino problem based on phenomena which are not the convention mass-induced neutrino oscillations is still viable. The ultimate goal is of course a direct experimental test of these solution. The upcoming solar neutrino experiments will provide a lot of new information which hopefully will reveal the true nature of the solar neutrino problem.

\section{References}

[1] T.B. Cleveland et al. (Homestake Collaboration), Astrophys. J. 496, 505 (1998).

[2] W. Hampel et al. (GALLEX Collaboration), Phys. Lett. B 447, 127 (1999)

[3] J.N. Abdurashitov et al. (SAGE Collaboration), Phys. Rev. C 60, 055801 (1999).

[4] Y. Fukuda et al. (Kamiokande Collaboration), Phys. Rev. Lett. 77, 1683 (1996).

[5] Y. Fukuda et al. (SuperKamiokande Collaboration), Phys. Rev. Lett. 82, 1810 (1999).

[6] J.N. Bahcall and M.H. Pinsonneault, Rev. Mod. Phys. 67, 781 (1995). 
[7] J.N. Bahcall, M.H. Pinsonneault, S. Basu and J. Christensen-Dalsgaard, Phys. Rev. Lett. 78, 171 (1997).

[8] J. N. Bahcall, S. Basu and M.H. Pinsonneault, Phys. Lett. B 433, 1 (1998).

[9] J.N. Bahcall, S. Basu and M.H. Pinsonneault, Phys. Lett. B 433, 1 (1998); see also J.N. Bahcall's home page, http://www.sns.ias.edu/ jnb.

[10] See also, J.N. Bahcall and R.K. Ulrich, Rev. Mod. Phys. 60, 297 (1988); J.N. Bahcall and M.H. Pinsonneault, Rev. Mod. Phys. 64, 885 (1992); S. TurckChieze et al., Astrophys. J. 335, 415 (1988); S. TurckChieze and I. Lopes, Astrophys. J. 408, 347 (1993); V. Castellani, S. Degl'Innocenti and G. Fiorentini, Astron. Astrophys. 271, 601 (1993); V. Castellani et al., Phys. Lett. B 324, 425 (1994).

[11] J.N. Bahcall, Neutrino Astrophysics, Cambridge University Press, 1989.

[12] E.G. Adelberger et al., Rev. Mod. Phys. 70, 1265 (1998).

[13] For a detailed list of the references on these solutions see: "Solar Neutrinos: The First Thirty Years", ed. by R. Davis Jr. et al., Frontiers in Physics, Vol. 92, Addison-Wesley, 1994.

[14] See for example:

D.O. Caldwell and R.N. Mohapatra, Phys. Rev. D 48, 3259 (1993); J. T. Peltoniemi, D. Tommasini, and J.W.F. Valle, Phys. Lett. B 298, 383 (1993); J.T. Peltoniemi and J.W.F. Valle, Nucl. Phys. B 406, 409 (1993); G. Dvali and Y. Nir, JHEP 9810, 014 (1998) [hep-ph/9810257]; G. M. Fuller, J. R. Primack and Y.Z. Qian, Phys. Rev. D52, 1288 (1995); J. J. GomezCadenas and M. C. Gonzalez-Garcia, Zeit. fur Physik C71, 443 (1996); N. Okada and O. Yasuda, Int. J. Mod. Phys. A 12, 3669 (1997), and references therein.

[15] V. N. Gribov and B. M. Pontecorvo, Phys. Lett. B 28, 493 (1969).

[16] L. Wolfenstein, Phys. Rev. D17, 2369 (1978).

[17] S.P. Mikheyev and A. Yu. Smirnov, Sov. J. Nucl. Phys. 42, 913 (1985); Nuovo Cimento C9, 17 (1986).

[18] J.N. Bahcall, P.I. Krastev and A.Yu. Smirnov, Phys. Rev. D 58, 096016 (1998) [hep-ph/9807216]; Phys. Rev. D 60, 093001 (1999) [hep-ph/9905220].

[19] M.C. Gonzalez-Garcia, P.C. de Holanda, C. PeñaGaray and J.W.F. Valle, Nucl. Phys. B 573 (2000) 3 [hep-ph/9906469]. Holanda

[20] G.L. Fogli, E. Lisi, D. Montanino and A. Palazzo, Phys. Rev. D 62 (2000) 0130032 [hep-ph/9912231].

[21] A. Cisneros, Astrophys. Space Sci. 10, 87 (1971).

[22] L. B. Okun, M. B. Voloshin, and M. I. Vysotsky, Sov. Phys. JETP 64, 446 (1986).

[23] J. Schechter and J. W. F. Valle, Phys. Rev. 24, 1883 (1981); ibid25, 283 (1982).

[24] C. S. Lim and W. J. Marciano, Phys. Rev. 37, 1368 (1988).

[25] E. Kh. Akhmedov, Sov. J. Nucl. Phys. 48, 382 (1988); Phys. Lett. B213, 64 (1988).
[26] For reviews, see for e.g., J. Pulido, Phys. Rep 211, 167 (1992); E. Kh. Akhmedov, talk presented at 4th International Solar Neutrino Conference, Heidelberg, Germany, April 1997, hep-ph/9705451 and references therein.

[27] E. Kh. Akhmedov, A. Lanza, and S. T. Petcov, Phys. Lett. B303, 85 (1993).

[28] P. I. Krastev, Phys. Lett. B303, 75 (1993).

[29] C. S. Lim and H. Nunokawa, Astropart. Phys. 4, 63 (1995).

[30] J. Pulido, Phys. Rev. D57, 7108 (1998).

[31] B. C. Chauhan, U. C. Pandey and S. Dev, Mod. Phys. Lett. A13, 1163 (1998).

[32] M.M. Guzzo and H. Nunokawa, Astrop. Phys. 12, 87 (1999).

[33] J. N. Bahcall, P. I. Krastev and E. Lisi, Phys. Rev. C55, 494 (1997).

[34] G. L. Fogli and E. Lisi, Astro. Part. Phys. 3, 185 (1995).

[35] H. Minakata and H. Nunokawa, Phys. Rev. D59, 073004 (1999), and references therein for the previous works.

[36] Note that, strictly speaking, such right handed muon (or tau) neutrinos can contribute to water Cherenkov detector though electromagnetic interaction provided that $\mu_{\nu}$ is large enough. However, such contribution is small enough to be neglected for the magnetic moment we are assuming in this work.

[37] J. Schmitt and R. Rosner, Astrophys. J. 265, 901 (1983).

[38] X. Shi et al., Comm. Nucl. Part. Phys. 21, 151 (1993).

[39] J. Pulido, hep-ph/9808319.

[40] S. Bergmann, M.M. Guzzo, P.C. de Holanda, P. Krastev and H. Nunokawa, Phys. Rev. D (2000) 073001 [hep-ph/0004049].

[41] M. M. Guzzo, N. Reggiani and J.H.Colonia, Phys. Rev. D56, 588 (1997); M. M. Guzzo, N. Reggiani, P. H. Sakanaka, Phys. Lett. B357, 602 (1995).

[42] G. Fiorentini, M. Moreti and F.L.Villante, Phys. Lett. B413, 378 (1997).

[43] S. Pastor, V.B.Semikoz and J.W.F.Valle, Phys. Lett. B423, 118 (1998).

[44] M.M. Guzzo, A. Masiero and S.T. Petcov, Phys. Lett. B 260, 154 (1991).

[45] V. Barger, R.J.N. Phillips and K. Whisnant, Phys. Rev. D 44, 1629 (1991).

[46] E. Roulet, Phys. Rev. D 44, 935 (1991).

[47] S. Degl'Innocenti and B. Ricci, Mod. Phys. Lett. A 8, 471 (1993).

[48] G.L. Fogli and E. Lisi, Astroparticle Phys. 2, 91 (1994).

[49] P.I. Krastev and J.N. Bahcall, "FCNC solutions to the solar neutrino problem", hep-ph/9703267.

[50] S. Bergmann, Nucl. Phys. B 515, 363 (1998) [hep-ph/9707398]. 
[51] Resonant neutrino conversion induced by nonorthogonal massless neutrinos was first discussed by J.W.F. Valle in Phys. Lett. B 199, 432 (1987). However this mechanism can not induce a large effect on the solar neutrinos due to the stringent constraints on the model parameters. See also Refs. [53, 54].

[52] S. Bergmann, Y. Grossman and E. Nardi, Phys. Rev. D 60, 093008 (1999) [hep-ph/9903517].

[53] P. Langacker and D. London, Phys. Rev. D 38, 886 (1988); ibid 38, 907 (1988); H. Nunokawa, Y.-Z. Qian, A. Rossi and J.W.F. Valle, Phys. Rev. D 54, 4356 (1996) [hep-ph/9605301], and references therein.

[54] S. Bergmann and A. Kagan, Nucl. Phys. B 538, 368 (1999) [hep-ph/9803305].

[55] C.S. Aulakh and N.R. Mohapatra, Phys. Lett. B 119, 136 (1983); F. Zwirner, Phys. Lett. B 132, 103 (1983); L.J. Hall and M. Suzuki, Nucl. Phys. B 231, 419 (1984); J. Ellis et al., Phys. Lett. B 150, 142 (1985); G.G. Ross and J.W.F. Valle, Phys. Lett. B 151, 375 (1985); R. Barbieri and A. Masiero, Phys. Lett. B 267, 679 (1986).

[56] S.P. Mikheyev and A.Yu. Smirnov, Sov. Phys. Usp. 30, 759 (1987).

[57] M. Freund and T. Ohlsson, hep-ph/9909501.

[58] E.Kh. Akhmedov, Nucl. Phys. B 538, 25 (1999).

[59] G.L. Fogli, E. Lisi and D. Montanino, Phys. Rev. D 49, 3226 (1994).

[60] Y. Suzuki, "Solar Neutrinos", talk given at the Lepton Photon Conference, 1999.

[61] See Fig. 8.2 of Ref. [11] for the recoil electron spectra shape from ${ }^{8} \mathrm{~B}$ neutrino due to $\nu_{e} e^{-} \rightarrow \nu_{e} e^{-}$and $\nu_{\mu, \tau} e^{-} \rightarrow \nu_{\mu, \tau} e^{-}$scattering.

[62] P.C.de Holanda, C.Peña-Garay, M.C.Gonzalez-Garcia and J.W.F.Valle, Phys. Rev. D 60 (1999) 093010.

[63] E. Ma and D. P. Roy, Phys. Rev. D 58, 095005 (1998) [hep-ph/9806210], E. Ma, Phys. Lett. B 433, 74 (1998) [hep-ph/9709474].
[64] L.J. Hall, V.A. Kostelecky, S. Raby, Nucl. Phys. B B267 415 (1986); Y. Okada, hep-ph/9809297.

[65] F. Pisano and V. Pleitez, Phys. Rev. D 46, 410 (1992); P. Frampton, Phys. Rev. Lett. 69, 2889 (1992).

[66] E. G. Adelberger et al., astro-ph/9805121, Rev. Mod. Phys., 70, 1265 (1998).

[67] E. Roulet, Phys. Rev. D44, 935 (1991).

[68] See for e.g, N. Hata and P. Langacker, Phys. Rev. D56, 6107 (1997); G. L. Fogli, E. Lisi and D. Montanino, Astropart. Phys. 9, 119 (1998); J. N. Bahcall, P. I. Krastev and A. Yu. Smirnov, in ref. [18].

[69] H. Minakata and H. Nunokawa, Phys. Rev. Lett. 63, 121 (1989); A. B. Balantekin, P. J. Hatchell and F. Loreti, Phys. Rev. D41, 3583 (1990). H. Nunokawa and H. Minakata, Phys. Lett. B314, 371 (1993); E. Kh. Akhmedov, A. Lanza, and S. T. Petcov, Phys. Lett. B348, 124 (1995).

[70] C. Caso et al., The European Physical Journal C3, 1 (1998).

[71] We note, however, that the more stringent bound, $\mu_{\nu}<3 \times 10^{-12} \mu_{B}$, is obtained from the argument of the cooling of the red giants, in G. Raffelt, Phys. Rev. Lett. 64, 2856 (1990).

[72] A. Gago, H. Nunokawa and R. Z. Funchal, Phys. Rev. Lett. 84, 4035 (2000).

[73] M. Gasperini, Phys. Rev. D 38, 2635 (1988); 39, 3606 (1989).

[74] For recent analysis, see e.g.: M.C. Gonzalez-Garcia, et al., D 58, 033004 (1998); M.C. Gonzalez-Garcia, H. Nunokawa, O.L.G. Peres and J.W.F. Valle, B 543, 3 (1999) [hep-ph/9807305]; N. Fornengo, M.C. GonzalezGarcia and J.W.F. Valle, hep-ph/0002147.

[75] C. Athanassopoulos et al. (LSND Collaboration), Phys. Rev. Lett. 77, 3082 (1996); Phys. Rev. Lett. 81, 1774 (1998). 